\title{
Physical property characterization of Fe-tube encapsulated and vacuum
}

\section{annealed bulk $\mathrm{MgB}_{2}$}

V.P.S. Awana ${ }^{1,2, \$}$, Rajeev Rawat ${ }^{3}$, Anurag Gupta ${ }^{1}$, M. Isobe ${ }^{2}$, K.P. Singh ${ }^{1}$, Arpita Vajpayee ${ }^{1}$, H. Kishan ${ }^{1}$, E. Takayama-Muromachi ${ }^{2}$ and A.V. Narlikar ${ }^{3}$

${ }^{1}$ National Physical Laboratory, Dr. K.S. Krishnan Marg, New Delhi-110012, India

${ }^{2}$ Superconducting Materials Center, NIMS, 1-1 Namiki, Tsukuba, Ibaraki, 305-0044, Japan

${ }^{3}$ UGC-DAE Consortium for Scientific Research, University Campus, Khandwa Road, Indore-452017, India

We report phase formation, and detailed study of magnetization and resistivity under magnetic field of $\mathrm{MgB}_{2}$ polycrystalline bulk samples prepared by Fe-tube encapsulated and vacuum (10 $0^{-5}$ torr) annealed $\left(750{ }^{0} \mathrm{C}\right)$ route. Zero-field-cooled magnetic susceptibility $\left(\chi^{\mathrm{ZFC}}\right)$ measurements exhibited sharp transition to superconducting state with a sizeable diamagnetic signal at $39 \mathrm{~K}\left(T_{c}\right)$. The measured magnetization loops of the samples, despite the presence of flux jumps, exhibited a stable current density $\left(J_{c}\right)$ of around $2.4 \times 10^{5} \mathrm{~A} / \mathrm{cm}^{2}$ in up to $2 \mathrm{~T}$ (Tesla) field and at temperatures $(T)$ up to $10 \mathrm{~K}$. The upper critical field is estimated from resistivity measurements in various fields and shows a typical value of $8 \mathrm{~T}$ at $21 \mathrm{~K}$. Further, $\chi^{\mathrm{FC}}$ measurements at an applied field of $0.1 \mathrm{~T}$ reveal paramagnetic Meissner effect $(P M E)$ that is briefly discussed.

Key Words: $\mathrm{MgB}_{2}$, Critical current density, and Magnetization \$: Corresponding Author: awana@ mail.nplindia.ernet.in 


\section{INTRODUCTION}

Soon after the discovery of superconductivity in $\mathrm{MgB}_{2}$ with the transition temperature of $\sim 40 \mathrm{~K}^{1}$, the focus changed within a year from basic to applied research in terms of improving the critical current density $\left(J_{c}\right)$ of the compound for practical purposes ${ }^{2-5}$. Clean $\mathrm{MgB}_{2}$ compounds having high residual resistivity ratio (RRR), defined as $R_{300 K} / R_{40 K}$ exhibited low critical current density $\left(J_{c}\right)$ values. This limited the scope of this compound for any viable practical use. However soon it was realized that induction of disorder could result in record high $J_{c}$ values with only a marginal reduction in $T_{c}^{3-6}$.

So far, a number of techniques have been developed to increase the $J_{c}$ of $\mathrm{MgB}_{2}$,: for example, (a) various nano-particle doping viz. nano-SiC ${ }^{3,4}$, carbon-nanotubes ${ }^{5}$ and nano-diamond ${ }^{6}$, and (b) following various heating schedules viz. liquid assisted sintering ${ }^{7}$ and combustion ${ }^{8}$. Similar novel methods had earlier been tried in case of high $T_{c}$ cuprate superconductors, but were not as successful as in the case of $\mathrm{MgB}_{2}$. The ultimate aim had been to create optimally distributed pinning centers in a superconductor to inhibit the motion of vortices and thereby achieve a high $J_{c}$. By now, via the various techniques of doping/substitutions or heating methodology, the $J_{c}$ values in excess of $10^{6} \mathrm{~A} / \mathrm{cm}^{2}$ have already been achieved for $\mathrm{MgB}_{2}{ }^{3-8}$. There is a race currently in progress to produce high quality $\mathrm{MgB}_{2}$ powder for making wires and tapes ${ }^{3-8}$ to carry higher transport current densities. In this regards, we report here $\mathrm{dc}$ susceptibility, magnetization and magnetoresistivity characterization of bulk polycrystalline $\mathrm{MgB}_{2}$ compounds produced by Fe-tube encapsulated and vacuum $\left(10^{-5}\right.$ torr $)$ annealed $\left(750{ }^{0} \mathrm{C}\right)$ route.

\section{EXPERIMENTAL}

Our $\mathrm{MgB}_{2}$ samples were synthesized by encapsulation of well mixed and palletized high quality (above $3 \mathrm{~N}$ purity) $\mathrm{Mg}$ and B powders with some added Mg turnings in a soft Fe-tube and its subsequent 
heating to $750{ }^{\circ} \mathrm{C}$ for two and half hours in an evacuated $\left(10^{-5}\right.$ Torr) quartz tube and quenching to liquidnitrogen temperature. Details of synthesis procedure are given elsewhere ${ }^{9,10}$. In our case the resultant sample was a bulk polycrystalline compound. The x-ray diffraction pattern of the compound was recorded with a diffractometer using $\mathrm{CuK}_{\alpha}$ radiation. Resistivity measurements are carried out by four-probe technique under applied field of up to 8 Tesla. Magnetization measurements were carried out with a Quantum-Design SQUID magnetometer (MPMS-XL).

Fig. 1 depicts the x-ray diffraction pattern of the currently studied Fe-tube encapsulated and vacuum ( $10^{-5}$ torr) annealed $\mathrm{MgB}_{2}$ polycrystalline bulk sample. The compound has hexagonal Bravais lattice with lattice parameters of $\mathrm{a}=3.086 \AA$, and $\mathrm{c}=3.524 \AA$. It is clear from this figure that the compound is nearly single phase, with small un-reacted line at around $2 \theta \cong 36^{\circ}$ and $63^{\circ}$. The one at $2 \theta \cong$ $36^{\circ}$ is due to a $\mathrm{Mg}$ metal ${ }^{11}$ and other at $63^{\circ}$ is due to presence of $\mathrm{MgO}$, as suggested earlier by various authors $^{4,11}$.

Resistivity versus temperature $\rho(T)$ plots of our $\mathrm{MgB}_{2}$ compound, are presented in Fig. 2 and Fig. 3. Namely $\rho(T)$ plots are shown in Fig. 2 and Fig.3 depicts the $\mathrm{d} \rho / \mathrm{d} T(T)$ plots in various applied fields of up to 8 Tesla. The extended part of $\rho(T)$ plots is further shown for transition region in inset of Fig. 2. Single $\mathrm{d} \rho / \mathrm{d} T(T)$ peaks for all measurements under various applied fields indicate towards good quality of the present sample. The superconducting transition temperatures $\left(T_{c}\right)$ are defined by the peak temperature of $\mathrm{d} \rho / \mathrm{d} T(T)$ plots, which in turn determine the upper critical fields $\left(H_{c 2}\right)$ at those temperatures. The plot of $H_{c 2}(T)$ being estimated with such a reasoning is shown in inset of Fig. 3. The upper critical field shows a typical value of $8 \mathrm{~T}$ at $21 \mathrm{~K}$.

Fig.4 depicts the dc susceptibility $(\chi)$ versus temperature plots for the $\mathrm{MgB}_{2}$ sample in an applied field of $5 \mathrm{Oe}$, in both zero-field-cooled (ZFC) and field-cooled (FC) situations. It is evident from this figure that the present $\mathrm{MgB}_{2}$ undergoes a sharp superconducting transition (diamagnetic) at around $39 \mathrm{~K}$ within less than $2 \mathrm{~K}$ temperature interval, without any rounding before saturation down to $5 \mathrm{~K}$. In fact, the 
diamagnetic signal remains more or less constant below $36 \mathrm{~K}$ down to $5 \mathrm{~K}$. The $\chi^{\mathrm{FC}}$ signal around $T_{c}$ is weak and indicates strong pinning in the samples. Interestingly, the $\chi^{\mathrm{FC}}(T)$ plot in higher applied field of $0.1 \mathrm{~T}$, as shown in the inset of Fig.4, exhibits a paramagnetic transition around $37 \mathrm{~K}$. This effect has been called Paramagnetic Meissner Effect $(P M E)$. The thermal cycling of $\chi^{\mathrm{FC}}(T)$ in terms of $F C C$ (FC-cooling) and $F C W\left(F C\right.$-warming) exhibits hysteresis ${ }^{12}$, as evident in inset of Fig. 4 , where $\chi^{\mathrm{FC}}(T)$ measurements are carried out in 0.1 Tesla field in both $F C C$ and $F C W$ situations. The details of $P M E$ phenomenon, shall be a subject of separate article. However, the presence of $P M E$ does imply that the sample must be containing intrinsic SIS (Superconductor-Insulator-Superconductor) or SNS (Superconductor-NormalSuperconductor) junctions ${ }^{12,13}$. It is argued earlier that pi-junctions of SIS/SNS in favorable conditions can give rise to PME. The presence of micron or smaller normal/insulating impurities, for instance $\mathrm{Mg} / \mathrm{MgO}$ (as seen in XRD, Fig.1) could act as possible SIS/SNS junction to favor the observed $P M E$ in the samples. Interestingly, these nano-metric impurities might very well act as effective pinning centers to give high $\mathrm{J}_{\mathrm{C}}$ in the samples (see below).

In Fig. 5 we show the magnetic hysteresis $M(H)$ loops for our $750{ }^{0} \mathrm{C}$ annealed $\mathrm{MgB}_{2}$ compound at 5, 10, 20, 25 and $30 \mathrm{~K}$ with applied fields $(H)$ of up to \pm 7 Tesla. As is evidenced from this figure, there are some interesting points to note that (i) despite the flux jumps, the magnetization of our samples is nearly invariant, for up to $T \leq 20 \mathrm{~K}$, in the field range of $1 \mathrm{~T}>H>-1 \mathrm{~T}$ and (ii) the irreversibility field $\left(H_{M}{ }^{*}\right)$, as defined in ref. 14 , are much lower than $H_{C 2}$ values, e.g., at $20 \mathrm{~K}$ the respective values are $4.5 \mathrm{~T}$ and 8.5 T. The $H_{M}{ }^{*}$ for our sample is depicted in inset I of Fig. 5. We estimated the $J_{c}$ of our sample by using Bean's critical state model. Our sample studied for magnetization was in a cylindrical form, hence we used the formula $J_{c}=30 \times \Delta M / d$. Where $\Delta M=\left|\mathrm{M}_{+}\right|-|\mathrm{M}| \mid$ comes from the measured $M(H)$ loops and $d$ is the diameter of the cylindrical sample. For our sample the stabilized $\Delta M$ has a value of around 1100 emu/cm $\mathrm{cm}^{3}$ within $1 \mathrm{~T}>H>-1 \mathrm{~T}$ at $5 \mathrm{~K}, 10 \mathrm{~K}$, and $20 \mathrm{~K}$. Taking $d=0.11 \mathrm{~cm}$, the value of $J_{c}$ comes out to be $2.4 \times 10^{5} \mathrm{~A} / \mathrm{cm}^{2}$ which seems to be a competitive value. Note that further stabilization of flux jumps 
can result in much higher $J_{c}$ values. The plots of $J_{c}$ as a function of $H$ at different values of $T$ are shown in the inset II of Fig. 5. As seen from the figure, at all temperatures, $J_{c}$ is found to decrease with applied field. The decrease gets more rapid with increasing $\mathrm{H}$ and $\mathrm{T}$. Further, we should mention that, the presence of flux jumps (the region marked in Fig.5) lowers the $\mathrm{J}_{\mathrm{C}}$ in our samples. Similar flux jumps at low temperatures have been earlier reported in doped $\mathrm{MgB}_{2}$ compounds ${ }^{16,17}$. The fluxoid jumps are supposed to occur only in case of extremely high critical currents and very low heat capacity, resulting in localized motion of magnetic flux ${ }^{18}$.

In conclusion, we have shown that bulk $\mathrm{MgB}_{2}$ produced by Fe-tube encapsulated and vacuum $\left(10^{-5}\right.$ torr) annealed $\left(750{ }^{\circ} \mathrm{C}\right)$ route has a competitive $J_{c}$ that is practically invariant up to $20 \mathrm{~K}$ within field range of $1 \mathrm{~T}>H>-1 \mathrm{~T}$. Stabilizing the flux jumps can further enhance the $J_{c}$ of these samples. The irreversibility field $H_{M}$ * is found to be much smaller than $H_{c 2}$. The observation of $P M E$ effect does point out the presence of micron or smaller normal/insulating impurities in the samples that may be responsible for high $J_{c}$ in the samples. However, further optimization of synthesis is required to increase the $H_{M}{ }^{*}$.

Authors from NPL appreciate the interest and advice of Prof. Vikram Kumar (Director) NPL in the present work. The work is partly supported by INSA-JSPS bilateral exchange program. 


\section{FIGURE CAPTIONS}

Fig. 1 X-ray diffraction pattern for $750{ }^{\circ} \mathrm{C}$ annealed $\mathrm{MgB}_{2}$ compound.

Fig. 2 Resistivity versus temperature $\rho(T)$ plots under various applied fields. The inset shows the extended $\rho(T)$ plots of the same.

Fig. $3 \mathrm{~d} \rho / \mathrm{d} T$ versus $T$ plots under various applied field. The inset shows the $H_{c 2}(T)$ plot.

Fig. 4 Dc magnetic susceptibility versus temperature $\chi(T)$ plot in both zero-field-cooled (ZFC) and field-cooled (FC) situations at $\mathrm{H}=5$ Oe. The inset shows the same for FC situation with $\mathrm{H}=1000$ Oe.

Fig. 5 Magnetic hysteresis $M(H)$ loops plots at 5,10,20 and $30 \mathrm{~K}$ with applied fields $(H)$ of up to \pm 7 Tesla. Inset-I shows the expanded $M(H)$ loops to mark the $H_{M}{ }^{*}$. Insets - II shows the $J_{c}$ ( $H$ ) plots. 


\section{REFERENCES}

1. J. Nagamatsu, N. Nakagawa, T. Muranaka, Y. Zenitani, and J. Akimitsu, Nature (London) 410 (2001) 63.

2. D.C. Larbalestier, M.O. Rikel, L.D. Cooley, A.A. Polyanskii, J.Y. Jiang, S. Patnaik, X.Y. Cai, D.M. Feldmann, A. Gurevichi, A.A. Squitieri, M.T. Naus, C.B. Eom, E.E. Hellstrom, R.J. Cava, K.A. Regan, N. Rogado, M.A. Hayward, T. He, J.S. Slusky, P. Khalihah, K. Inumaru, and M. Hass, Nature (London) 410 (2001) 186.

3. S.X. Dou, S. Soltanian, X.L. Wang, P. Munroe, S.H. Zhou, M. Ionescu, H.K. Liu, and M. Tomsic, Appl. Phys. Lett. 81 (2002) 3419.

4. A. Matsumoto, H. Kumakura, H. Kitaguchi, and H. Hatakeyama, Sup. Sci. and Tech. 16 (2003) 926.

5. S.X. Dou, W.K. Yeoh, J. Horvat and M. Ionescu, Appl. Phys. Lett. 83 (2003) 4993.

6. C.H. Cheng, H. Zhang, Y. Zhao, Y. Feng, X.F. Rui, P. Munroe, H.M. Zheng, N. Koshizuka, and M. Murakami, Sup. Sci. and Tech. 16 (2003) 1182.

7. S.K. Chen, Z. Lockman, M. Wei, B.A. Glowacki, and J.L. MacManus-Driscoll, Appl. Phys. Lett. 86 (2005) 242501.

8. Y. Takano, N. Oguro, Y. Kaieda, K. Togano, Physica C 412-414 (2004) 125.

9. K. P. Singh, V.P.S. Awana, Md. Shahabuddin, R.B. Saxena, Rashmi Nigam, M.A. Ansari, Anurag Gupta, Himanshu Narayan, S. K. Halder, and H. Kishan to appear in Mod. Phys. Lett. B (2006).

10. R. Lal, V.P.S. Awana, K. P. Singh, R.B. Saxena, H. Kishan and A.V. Narlikar to appear in Mod. Phys. Lett. B (2006). 
11. Y. Zhu, L. Wu, V. Volkov, Q. Li, G. Gu, A.R. Moodenbaugh, M. Malac, M. Suenaga, and J. Tranquada, Physica C 356 (2001) 239.

12. W. Braunisch, N. Knauf, V. Kataev, S. Newhausen, R. Grutz, B. Roden, D. Khomskii, and D. Wohlleben, Phys. Rev. Lett. 68 (1992) 1908.

13. L. Pust, L.E. Wenger, and M.R. Koblischka, Phys. Rev. B 58 (1998) 14191.

14. S.X. Dou, V. Braccini, S. Soltanian, R. Klie, Y. Zhou, S. Li, X.L. Wang, and D. Larbalestier, J. Appl. Phys. 96 (2004) 7549.

15. A. Junod, Y. Wang, F. Bouquet and P. Toulemonde, Studies of High Temperature Superconductors,(Ed. A.V. Narlikar), Nova Sci., NY Vol. 38, p.179-205 (2002) Nova Sci., NY.

16. Gaojie J. Xu, J. -C. Grivel, A.B. Abrahmsen, and N.H. Andersen, Physica C 406 (2004) 95.

17. Victor Chabanenko, Roman Puzniak, Adam Nabialek, Sergei Vasiliev, Vladimir Rusakov, Loh Huanqian, Ritta Szymczak, Henryk Szymczak, Jan Juan, Jansuz Karpiniski, and Vitaly Finkel, J. Low Temp. Phys. 130 (2003) 175.

18. M. Tinkham, Introduction to Superconductivity 185, McGraw-Hill, New York, 1975. 
Fig. 1 Awana et al.

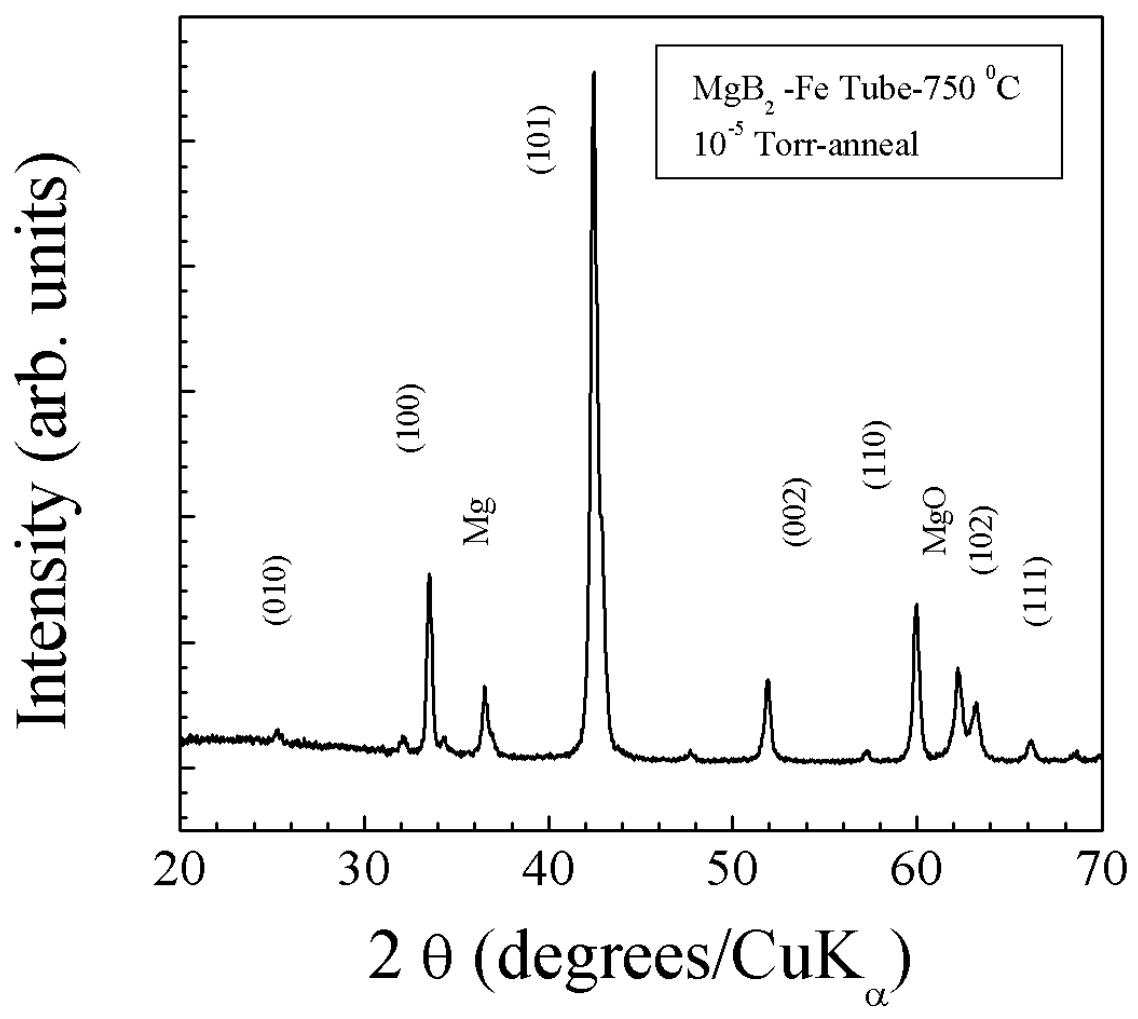


Fig. 2 Awana et al.

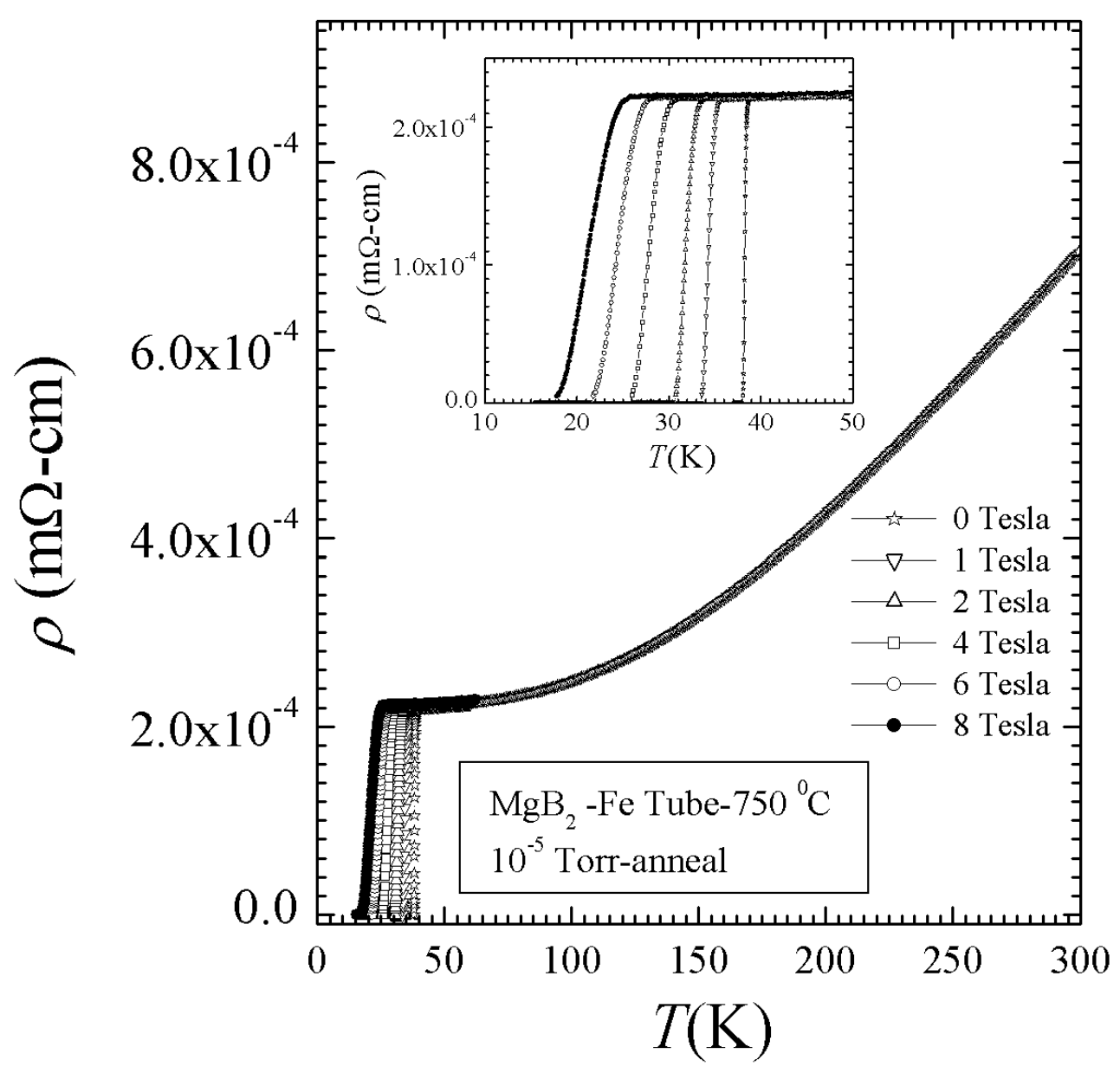


Fig. 3 Awana et al

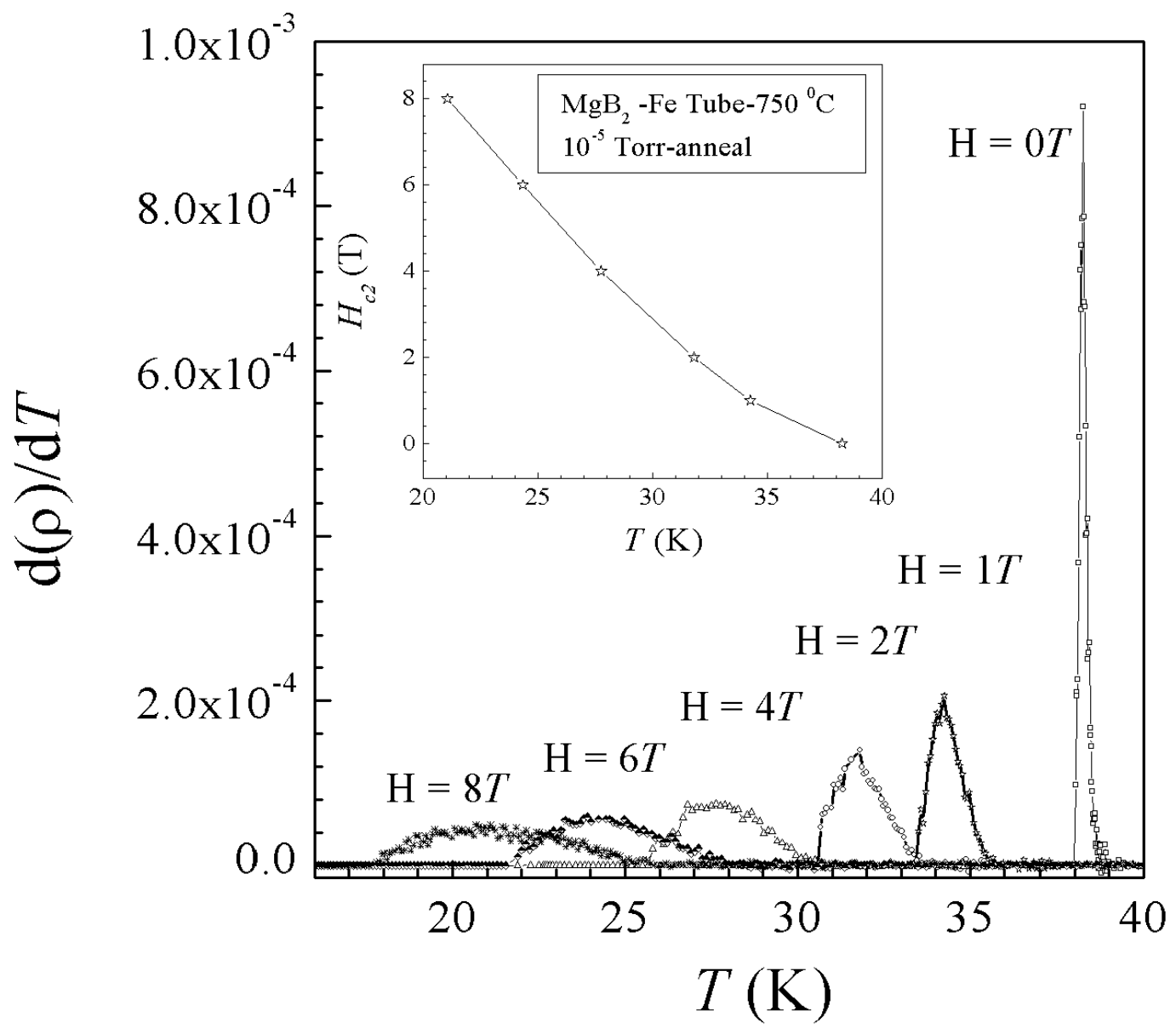


Fig. 4 Awana et al.

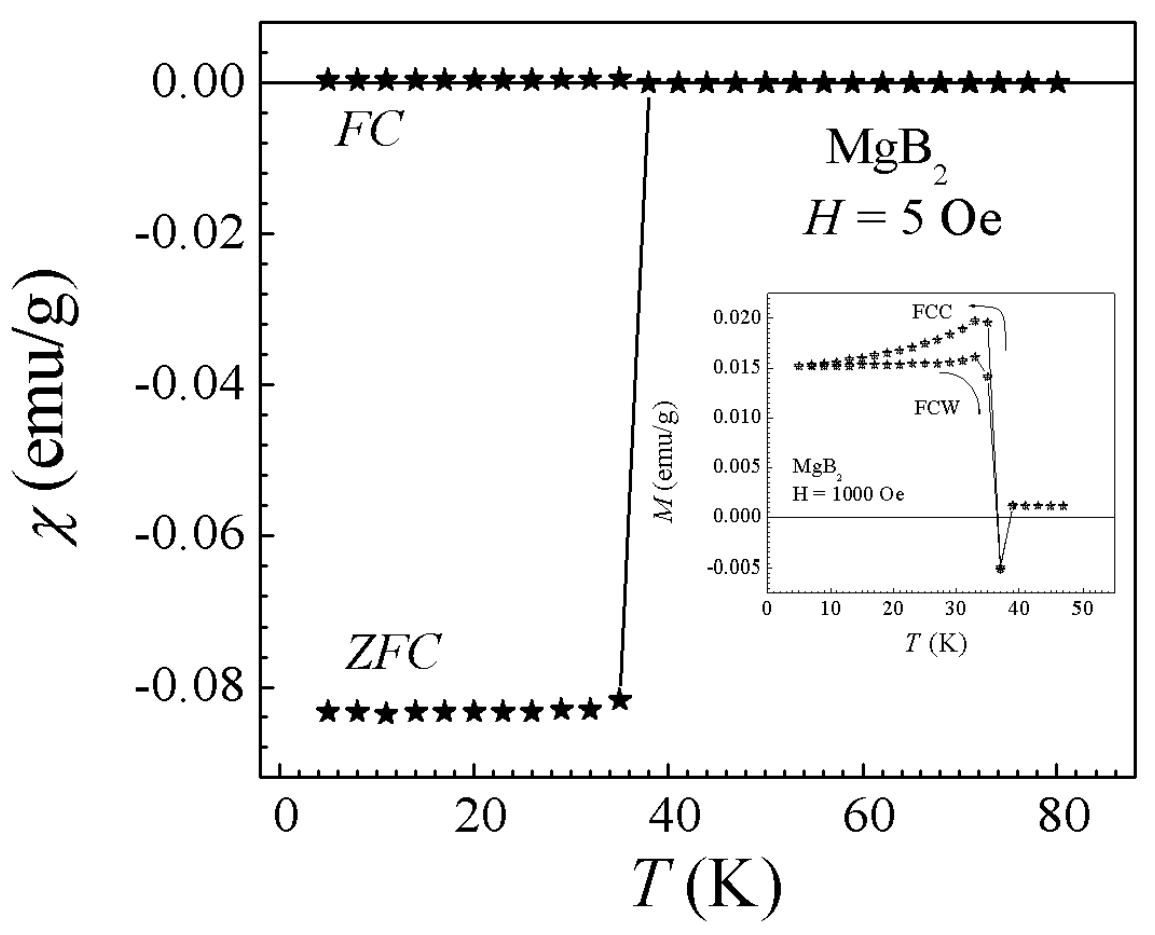


Fig. 5 Awana et al.

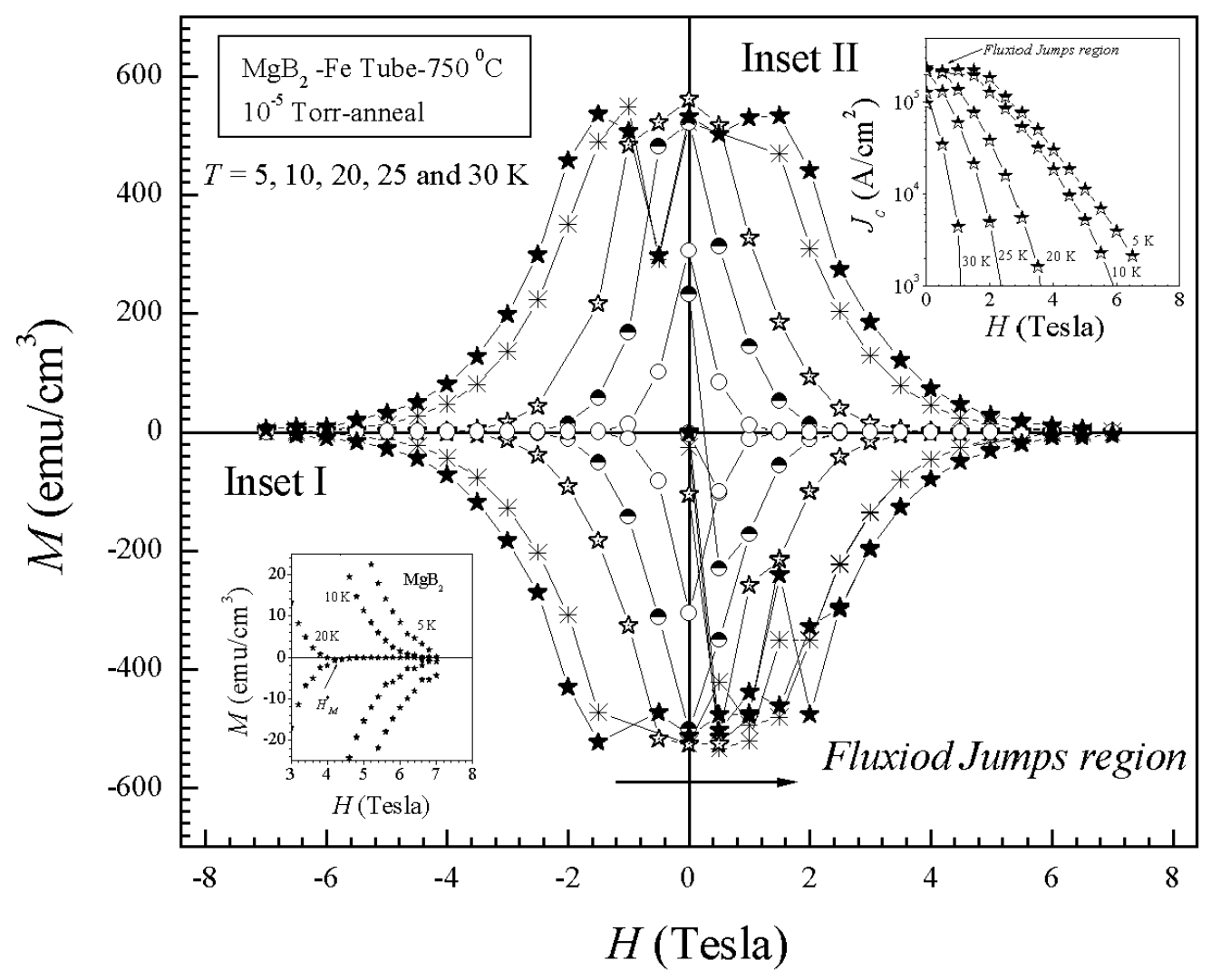

